Pré-Publicações do Departamento de Matemática

Universidade de Coimbra

Preprint Number 07-15

\title{
A LOGIC OF IMPLICATIONS IN ALGEBRA AND COALGEBRA
}

\author{
JIŘÍ ADÁMEK, MANUELA SOBRAL AND LURDES SOUSA
}

\begin{abstract}
Implications in a category can be presented as epimorphisms: an object satisfies the implication iff it is injective w.r.t. that epimorphism. G. Roçu formulated a logic for deriving an implication from other implications. We present two versions of implicational logics: a general one and a finitary one (for epimorphisms with finitely presentable domains and codomains). In categories $\operatorname{Alg} \Sigma$ of algebras on a given signature our logic specializes to the implicational logic of $\mathrm{R}$. Quackenbush. In categories Coalg $H$ of coalgebras for a given accessible endofunctor $H$ of sets we derive a logic for implications in the sense of P. Gumm.
\end{abstract}

\section{Introduction}

It has been observed by Bernard Banaschewski and Horst Herrlich [8] that implications in universal algebra are nothing else than injectivity w.r.t. regular epimorphisms $e$ in the category. Recall that an object $A$ is injective w.r.t. $e$ iff $\operatorname{hom}(A,-)$ turns $e$ into an isomorphism. Later Grigore Roçu [21] presented a logic of injectivity (= orthogonality ) w.r.t. epimorphisms in a category: the aim is to characterize, for a class $\mathcal{E}$ of epimorphisms, all injectivity consequences, i.e., all epimorphisms $e$ such that an object is $e$-injective whenever it is $f$-injective for every $f \in \mathcal{E}$.

In the present paper we formulate

(a) a deduction system for injectivity consequences

and

(b) the finitary variation where, as one does in universal algebra, only epimorphisms whose domains and codomains are finitely presentable are considered.

Received April 14, 2007.

The authors acknowledge financial assistance by Centre for Mathematics of University of Coimbra/FCT and International Center for Mathematics.

The first author acknowledges financial support by the Ministry of Education of the Czech Republic, Project MSM 6840770014.

The third author also acknowledges financial support by School of Technology of Viseu. 
Both are just minor variations of the deduction system of [21]. We prove that in every cocomplete and cowellpowered category our deduction systems are sound and complete. Both proofs are easy, and follow from results on injectivity classes presented in [18] and [23]. We then apply our deduction system to the category $\operatorname{Alg} \Sigma$ of algebras of a signature $\Sigma$ : if $\Sigma$ is finitary, the obtained logic of implications is that formulated by Robert Quackenbush [20], the infinitary variation is completely analogous. We also apply our deduction system to Coalg $H$, the category of coalgebras for an endofunctor $H$ of Set. Assuming that $H$ is $k$-accessible (i.e., preserves $k$-filtered colimits), covarieties of coalgebras can be presented by subsets of $C(k)$, a cofree coalgebra on $k$ colors, see [22]. The satisfaction of an implication

$$
M \Rightarrow N \quad \text { for } N \hookrightarrow M \hookrightarrow C(k)
$$

by a given coalgebra $A$ then means that for any coloring $f: A \rightarrow k$ by $k$ colors, the corrresponding homomorphism $f^{\sharp}: A \rightarrow C(k)$ fulfils

$$
f^{\sharp}[A] \subseteq M \quad \text { implies } \quad f^{\sharp}[A] \subseteq N .
$$

We formulate a deduction system for such implications which is sound and complete.

Acknowledges Discussions with Michel Hébert improved the presentation of our paper.

\section{Logic of Injectivity}

2.1. Definition An epimorphism $e: P \rightarrow Q$ is said to be an injectivity consequence of a set $\left\{e_{i}\right\}_{i \in I}$ of epimorphisms provided that every object $A$ injective w.r.t. $e_{i}$ for all $i \in I$ is also injective w.r.t. $e$. Notation:

$$
\left\{e_{i}\right\}_{i \in I}=e
$$


2.2. Injectivity Deduction System consists of one axiom

IDENTITY

$$
\overline{\operatorname{id}_{A}}
$$

and the following four deduction rules:

$$
\begin{aligned}
& \text { COMPOSItion } \frac{e, e^{\prime}}{e^{\prime} \cdot e} \text { if the codomain of } e \text { is the domain of } e^{\prime} \\
& \text { CANCELLATION } \frac{e^{\prime} \cdot e}{e} \\
& \text { PUSHOUT }
\end{aligned}
$$

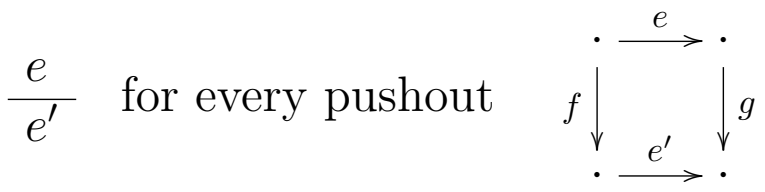

$$
\begin{aligned}
& \begin{array}{lll}
\text { COINTERSECTION } & \frac{e_{i}(i \in I)}{e} & \begin{array}{l}
\text { for every cointersection } e \\
\text { of } e_{i}: P \rightarrow Q_{i}(i \in I)
\end{array}
\end{array}
\end{aligned}
$$

2.3. Notation Let $\mathcal{E}$ be a class of epimorphisms of a category $\mathcal{A}$. We use

$$
\mathcal{E} \vdash e
$$

to denote the fact that $e$ can be proved from $\mathcal{E}$ by using the Injectivity Deduction System. That is, there exists a list $e_{1}, e_{2}, \cdots, e_{n}$ of morphisms for some cardinal $n$ such that $e_{n}=e$ and, for every $i<n, e_{i}$ is in $\mathcal{E}$ or $e_{i}$ is the conclusion of one of the above rules such that the assumptions are of the form $e_{j}$ for $j<i$.

2.4. Lemma The deduction system 2.2 is sound, i.e., $\mathcal{E} \vdash e$ implies $\mathcal{E} \models e$.

Proof (1) identity, COMPosition and CANCELlation are obvious.

(2) Pushout: Let $X$ be $e$-injective and let $f$ be an arbitrary morphism from the domain of $e^{\prime}$ to $X$ 


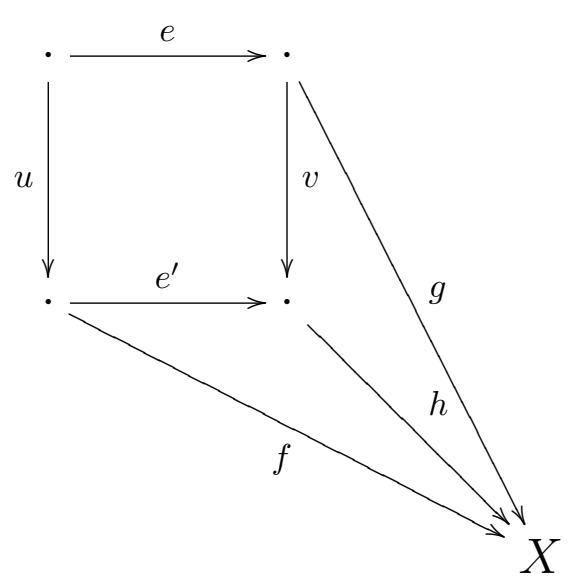

The $e$-injectivity of $X$ yields $g$ with $g e=f u$, and the universal property yields $h$ with $f=h e^{\prime}$.

(3) The proof of COINTERSECTION is analogous.

2.5. Theorem In every cocomplete cowellpowered category the deduction system 2.2 is complete: for any set $\mathcal{E}$ of epimorphisms $\mathcal{E} \models e$ implies $\mathcal{E} \vdash e$.

Proof We denote by $\tilde{\mathcal{E}}$ the class of all morphisms provable from $\mathcal{E}$. Given an injectivity consequence $e: P \rightarrow Q$ of $\mathcal{E}$, we prove $e \in \tilde{\mathcal{E}}$. Let $f: P \rightarrow$ $R$ be the cointersection of all members of $\tilde{\mathcal{E}}$ with domain $P$, then $f \in \tilde{\mathcal{E}}$ by Cointersection. From 2.5 in [23], $f$ is a reflection of $P$ in the full subcategory of $\mathcal{A}$ of all $\mathcal{E}$-injective objects. Thus, $f=g \cdot e$ for some $g: Q \rightarrow R$, then CANCELLATION implies $e \in \tilde{\mathcal{E}}$.

2.6. Remark We now turn to the finitary logic. Recall that an object $A$ is finitely presentable if the hom-functor $\operatorname{hom}(A,-)$ preserves filtered colimits. In the finitary logic we work with epimorphisms $e: P \rightarrow Q$ which are finitary by which we mean that $P$ and $Q$ are both finitely presentable. Example: as proved in [8] in universal algebra implications represented by regular epimorphisms precisely correspond to satisfaction of the formulas of the form

$$
\bigwedge_{i \in I}\left(e_{i}=e_{i}^{\prime}\right) \Rightarrow \bigwedge_{j \in J}\left(f_{j}=f_{j}^{\prime}\right)
$$

(where $e_{i}=e_{i}^{\prime}$ and $f_{j}=f_{j}^{\prime}$ are equations). And implications represented by finitary regular epimorphisms precisely correspond to the satisfaction of the first-order formulas as above, in other words, to the case where $I$ and $J$ 
are finite sets. Observe that the formula is then equivalent to finitely many formulas $\bigwedge\left(e_{i}=e_{i}^{\prime}\right) \Rightarrow\left(f=f^{\prime}\right)$.

We are going to formulate a finitary logic for deriving injective w.r.t. finitary morphisms. This is a variation of the logic presented by G. Roşu: the rules, and also the assumptions for proving the completeness, are slightly different. We use ideas of the classical work [11] of Gabriel and Zisman on the calculus of fractions, as exploited by Hébert, Adámek and Rosický in [18]. We start by recalling that concept.

2.7. Definition A class $\mathcal{E}$ of morphisms in a category $\mathcal{A}$ is said to admit a left calculus of fractions provided that

(i) $\mathcal{E}$ contains all identity morphisms,

(ii) $\mathcal{E}$ is closed under composition,

(iii) for every span

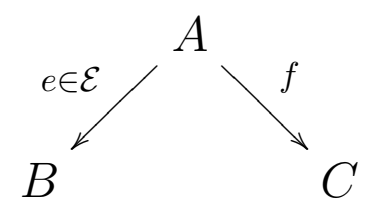

there exists a commutative square

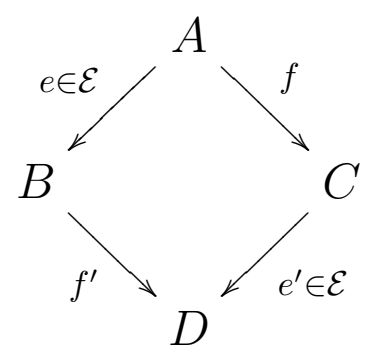

and

(iv) given parallel morphisms $h_{1}, h_{2}: B \rightarrow C$ in $\mathcal{A}$ such that $h_{1} e=h_{2} e$ for some morphism $e \in \mathcal{E}$ then there exists $e^{\prime} \in \mathcal{E}$ with $e^{\prime} h_{1}=e^{\prime} h_{2}$.

2.8. Remark (a) In particular, whenever a category has pushouts, every class $\mathcal{E}$ of epimorphisms containing all identity morphisms and closed under composition and pushout admits a left calculus of fractions.

(b) If $\mathcal{E}$ is a class of epimorphisms, the condition (iv) can be omitted: from $h_{1} e=h_{2} e$ we conclude $h_{1}=h_{2}$.

2.9. Notation Given a category $\mathcal{A}$, we denote by $\mathcal{A}_{f p}$ a full subcategory representing up to isomorphism all finitely presentable objects. 
2.10. Proposition (see [18]) Let $\mathcal{A}$ be a cowellpowered category with colimits, and let $\mathcal{E}$ be a class of epimorphisms of $\mathcal{A}_{f p}$ admitting a left calculus of fractions in $\mathcal{A}_{f p}$. Then every finitely presentable object $A$ in $\mathcal{A}$ has an $\mathcal{E}$ injective reflection $r_{A}: A \rightarrow A^{*}$ obtained by a filtered colimit of all morphisms $e: A \rightarrow X$ of $\mathcal{E}$ with $X \in \mathcal{A}_{f p}$.

More detailed: let $A \downarrow \mathcal{E}$ be the full subcategory of the comma category $A \downarrow \mathcal{A}_{f p}$ (of all morphisms with domain $A$ and codomain in $\mathcal{A}_{f p}$ ) formed by members of $\mathcal{E}$. Then the diagram

$$
D_{A}: A \downarrow \mathcal{E} \rightarrow \mathcal{A}, e \mapsto X
$$

is filtered, and if $A^{*}$ is the colimit of $D_{A}$ with the colimit cone $e^{*}: X \rightarrow A^{*}$ (for $e: A \rightarrow X$ in $A \downarrow \mathcal{E}$ ) then $r_{A}=i d_{A}^{*}$ is the reflection of $A$ in the full subcategory of $\mathcal{A}$ of all $\mathcal{E}$-injective objects. This means that $A^{*}$ is $\mathcal{E}$-injective, and given a morphism $f: A \rightarrow B$ such that $B$ is $\mathcal{E}$-injective, then $f$ factorizes through $r_{A}$. This was proved in [18] assuming that $\mathcal{A}$ is a finitely accessible category. But finite accessibility was only used to make the diagram $D_{A}$ essentially small. Since in the present paper $\mathcal{E}$ is a class of epimorphisms, this follows from $\mathcal{A}$ being cowellpowered.

2.11. Remark The above proposition implies that for finitary epimorphisms the deduction system 2.2 minus the last (nonfinitary) deduction rule COINTERSECTION gives a sound and complete logic:

2.12. Definition The Finitary Injectivity Deduction System is the deduction system of

IDENTITY, COMPOSITION, CANCELLATION and PUSHOUT in 2.2.

2.13. Finitary Completeness Theorem In every cocomplete cowellpowered category the Finitary Injectivity Deduction System is complete for sets $\mathcal{E}$ of finitary epimorphisms: whenever a finitary epimorphism $e$ is an injectivity consequence of $\mathcal{E}$, then $e$ has a (finite) proof from $\mathcal{E}$.

Proof Let $\overline{\mathcal{E}}$ denote the set of all epimorphims $e$ in $\mathcal{A}_{f p}$ such that $e$ has a finite proof from $\mathcal{E}$. Clearly $\overline{\mathcal{E}}$ contains all identity morphisms and is closed under composition and pushout. By 2.8(a), $\overline{\mathcal{E}}$ satisfies the calculus of fractions. By $2.4, \mathcal{E}$-injectivity implies $\overline{\mathcal{E}}$-injectivity.

Given a logical consequence $e_{0}: A \rightarrow B$ of $\mathcal{E}$ in $\mathcal{A}_{f p}$, let $r_{A}: A \rightarrow A^{*}$ be the $\overline{\mathcal{E}}$-injective reflection of 2.10. Since $A^{*}$ is $e_{0}$-injective, we have a morphism $f$ with $r_{A}=f \cdot e_{0}$. 
Since $B$ is finitely presentable, $\operatorname{hom}(B,-)$ preserves the filtered colimit $A^{*}=\operatorname{Colim}_{A}$. Thus, $f$ factorizes as $f=e^{*} \cdot f^{\prime}$, for some colimit morphism $e^{*}: X \rightarrow A^{*}$ of $D_{A}$ :

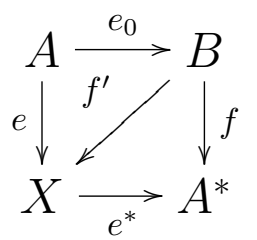

The diagram $D_{A}$ is filtered and the colimit morphism $e^{*}: X \rightarrow A^{*}$ merges the pair $e, f^{\prime} \cdot e_{0}: A \rightarrow X$. Since $A$ is finitely presentable, this implies the existence of an object of $A \downarrow \overline{\mathcal{E}}$ (i.e., a morphism $d: A \rightarrow Y$ in $\overline{\mathcal{E}}$ ) and a morphism $g: X \rightarrow Y$ such that $g \cdot e=d$ and $g$ also merges the above pair. Hence $g \cdot f^{\prime} \cdot e_{0}=d$, and then

$$
E \vdash d \text { implies } E \vdash e_{0},
$$

by CANCELLATION.

2.14. Remark (i) Our assumptions differ from those used by G. Roşu in [21] for his completeness theorem: he only required the morphism $e_{0}: A \rightarrow B$ to be finitely presentable as an object of the comma category $A \downarrow \mathcal{A}$, which is strictly weaker than our assumption that $e_{0}$ and all morphisms of $\mathcal{E}$ have finitely presentable domains and codomains. However G. Roşu assumed that the domains of his epimorphisms are projective, which is a strong assumption that our intended application (to quasivarieties) does not fulfill. Furthermore, instead of composition in $2.8 \mathrm{G}$. Roşu uses "union" stating that a pushout of two morphisms derived from $\mathcal{E}$ is derived from $\mathcal{E}$. This follows clearly from Pushout and Composition in 2.2. On the other hand, our formulation of the pushout rule is somewhat more restrictive than that used by G. Rosu : in applications of the above rule Pushout, $e$ is any morphism derived from $\mathcal{E}$, whereas Roşu's rule works with $e \in \mathcal{E}$.

(ii) Every cowellpowered category with colimits has a factorization system (epi, strong mono), see [3]. Theorem 2.13 can be generalized to any cocomplete and $E$-cowellpowered category with a factorization system $(E, M)$, which is the approach taken in [21].

2.15. Example Here we demonstrate that in Completeness Theorem 2.13 we cannot weaken the assumptions that the domains and codomains of morphisms $\mathcal{E} \cup\left\{e_{0}\right\}$ be finitely presentable to the assumption, used in [21], that these morphisms are finitely presentable. Recall from [17] that a morphism 
$f: P \rightarrow Q$ is finitely presentable if it is finitely presentable as an object of the slice category $P \downarrow A$.

In fact, in the category $\operatorname{Alg}(\Sigma)$, where $\Sigma$ consists of nullary symbols $a_{n}, n \in$ $\mathbb{N}$, we exhibit finitely presentable epimorphisms $\mathcal{E} \cup\{e\}$ with

$$
\mathcal{E} \models e \text { but } \mathcal{E} \nvdash e \text {. }
$$

A $\Sigma$-algebra $A$ is finitely presentable iff

(i) all but finitely many elements of $A$ have the form $a_{n}^{A}$, for some $n \in \mathbb{N}$ and

(ii) there are only finitely many pairs $m \neq n$ with $a_{m}^{A} \neq a_{n}^{A}$.

An epimorphism $h: A \rightarrow B$ in $\operatorname{Alg}(\Sigma)$ is finitely presentable iff there are only finitely many pairs $m, n$ with $a_{m}^{A} \neq a_{n}^{A}$ and $a_{m}^{B}=a_{n}^{B}$.

Denote by 1 the terminal $\Sigma$-algebra, by $I=\left\{a_{0}, a_{1}, a_{2}, \cdots\right\}$ the initial $\Sigma$ algebra and by $C$ the algebra $C=\{0,1\}$ having $a_{0}^{C}=0$ and $a_{i}{ }^{C}=1$ for all $i \geq 1$.

Let

$$
e_{0}: C \rightarrow 1
$$

be the trivial epimorphism and for every $k \geq 1$ define the quotient

$$
e_{k}: I \rightarrow I_{k}=I / \sim_{k}
$$

of $I$ modulo the least congruence $\sim_{k}$ with $a_{k}$ congruent to $a_{k+1}$.

For

$$
\mathcal{E}=\left\{e_{0}, e_{1}, e_{2}, \cdots\right\}
$$

an algebra is $\mathcal{E}$-injective iff all constants of $\Sigma$ in it are equal. Thus if

$$
e: I \rightarrow I / \approx
$$

denotes the quotient modulo the least congruence with $a_{0} \approx a_{1}$, we have that $\mathcal{E} \models e$.

We will prove that $\mathcal{E} \nvdash e$ by finding a set $\overline{\mathcal{E}}$ of epimorphisms with

$$
\left\{i d_{A} \mid A \in A \lg (\Sigma)\right\} \cup \mathcal{E} \subseteq \overline{\mathcal{E}} \quad \text { but } e \notin \overline{\mathcal{E}}
$$

which is closed under pushout, composition and left cancellation. This proves that $\overline{\mathcal{E}}$ contains all consequences of $\mathcal{E}$, thus $\mathcal{E} \nvdash e$.

Let $\overline{\mathcal{E}}$ be the set of all epimorphisms $g: B \rightarrow B^{\prime}$ such that

(1) $g$ is a finitely presentable morphism,

(2) if $g(x)=g\left(x^{\prime}\right)$ and $x \neq x^{\prime}$ then $x=a_{i}^{B}$ and $x^{\prime}=a_{j}^{B}$ for some $i, j$, and 
(3) if $B$ is finitely presentable and $a_{0}^{B} \neq a_{j}^{B}$ for all $j \geq 1$, then $a_{0}^{B^{\prime}} \neq a_{j}^{B^{\prime}}$ for all $j \geq 1$.

It is clear that $\overline{\mathcal{E}}$ contains $\left\{i d_{A} \mid A \in A \lg (\Sigma)\right\} \cup \mathcal{E}$ but it does not contain $e$ (since $e$ does not fulfil (3)).

a. $\overline{\mathcal{E}}$ is closed under pushout. In fact, let

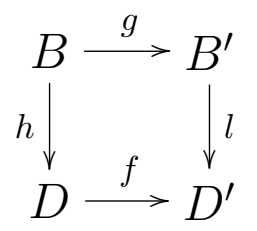

be a pushout with $g \in \overline{\mathcal{E}}$. It is easy to see that, since $g$ is finitely presentable, so is $f$. Thus it remains to verify (2) and (3). By the description of pushouts (in Set, hence in $A l g(\Sigma)$ ) $f$ merges a pair of elements $x \neq x^{\prime}$ of $D$ iff there exist the following zig-zag of elements $x=x_{0}, \cdots, x_{n+1}=x^{\prime}$ of $D$ and elements $y_{0}, \cdots, y_{2 n+1}$ of $B$

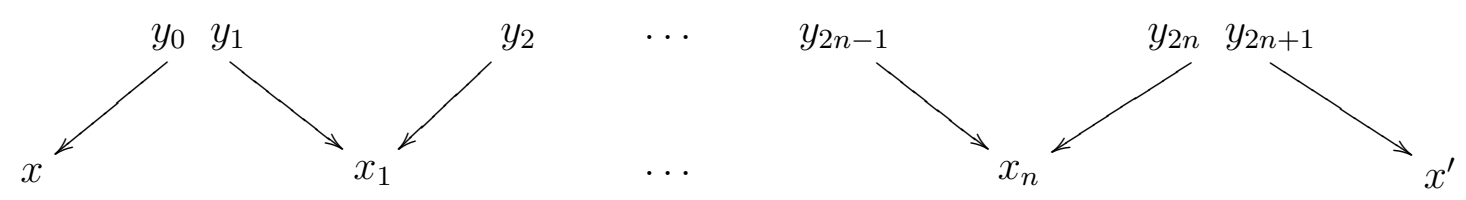

such that $x_{k}=h\left(y_{2 k-1}\right)=h\left(y_{2 k}\right)$ for $k=1, \ldots, n$, and $g\left(y_{2 k}\right)=$ $g\left(y_{2 k+1}\right)$, with $y_{2 k} \neq y_{2 k+1}$, for all $k=0, \cdots, n$.

To prove (2), let $f(x)=f\left(x^{\prime}\right)$ with $x \neq x^{\prime}$ and let us choose a zig-zag as above. Since $g$ fulfils (2), the equality $g\left(y_{0}\right)=g\left(y_{1}\right)$ implies $y_{0}=a_{i}^{B}$ for some i, thus $x=h\left(y_{0}\right)=a_{i}^{D}$. Analogously with $x^{\prime}$.

To prove (3), we assume that there exists a $k \geq 1$ with $a_{0}^{D^{\prime}}=a_{k}^{D^{\prime}}$, but $a_{0}^{D} \neq a_{j}^{D}$, for all $j \geq 1$, then we verify that $D$ is not finitely presentable. We have $a_{0}^{B} \neq a_{j}^{B}$, for all $j \geq 1$, and we will prove that $a_{0}^{B^{\prime}}=a_{l}^{B^{\prime}}$ for some $l \geq 1$ : it then follows that $B$ is not finitely presentable, since $g$ fulfils (3). Consequently, there exist infinitely many pairs $(u, v)$ with $a_{u}^{B}=a_{v}^{B}$ but $u \neq v$. For each such pair we have $a_{u}^{D}=a_{v}^{D}$, thus $D$ is not finitely presentable.

b. $\overline{\mathcal{E}}$ is closed under composition. In fact, given two composable morphisms

$$
B \stackrel{g}{\longrightarrow} B^{\prime} \stackrel{g^{\prime}}{\longrightarrow} B^{\prime \prime}
$$

in $\overline{\mathcal{E}}$ it is easy to see that $g^{\prime} \cdot g$ is finitely presentable. It fulfils (2) because, given $g^{\prime}(g(x))=g^{\prime}\left(g\left(x^{\prime}\right)\right)$ and $x \neq x^{\prime}$, then either $g(x)=$ 
$g\left(x^{\prime}\right)$ and we apply (2) to $g$, or $g(x) \neq g\left(x^{\prime}\right)$ and then (2) applied to $g^{\prime}$ yields $g(x)=a_{i}^{B}$ and $g\left(x^{\prime}\right)=a_{j}^{B}$, and then, again, we apply (2) to $g$. Finally, $g^{\prime} \cdot g$ fulfils (3): assume

$$
a_{0}^{B^{\prime \prime}}=a_{k}^{B^{\prime \prime}} \text { for some } k \text {, but } a_{0}^{B} \neq a_{j}^{B} \text { for all } j \geq 1 .
$$

If $a_{0}^{B^{\prime}}=a_{l}^{B^{\prime}}$ for some $l \geq 1$, then $B$ is not finitely presentable due to (3) applied to $g$. If $a_{0}^{B^{\prime}} \neq a_{l}^{B^{\prime}}$ for all $l \geq 1$ then $B^{\prime}$ is not finitely presentable, due to (3) applied to $g^{\prime}$. The latter implies again that $B$ is not finitely presentable: recall that $g: B \rightarrow B^{\prime}$ is a finitely presentable morphism, thus, $B$ is a finitely presentable object iff $B^{\prime}$ is one.

c. $\overline{\mathcal{E}}$ is closed under left cancellation. Given

$$
B \stackrel{g}{\longrightarrow} B^{\prime} \stackrel{g^{\prime}}{\longrightarrow} B^{\prime \prime}
$$

with $g^{\prime} \cdot g$ in $\overline{\mathcal{E}}$, then $g$ clearly fulfils (1) and (2). It fulfils (3) because $g^{\prime} \cdot g$ does.

\section{Implications in Algebra}

3.1. Assumption $\Sigma$ denotes a finitary, one-sorted signature. We assume a fixed countable set $V$ of variables. A free $\Sigma$-algebra on a set $W \subseteq V$ is denoted by $\phi(W)$. We are going to show that the Finitary Deduction System 2.12 is equivalent to the logic of implications presented by Robert Quackenbush [20].

Recall that an equation is a pair of elements of $\phi(V)$, notation: $u=v$. An implication is a formal expression

$$
\mathcal{P} \Rightarrow u=v
$$

where $\mathcal{P}$ is a finite set of equations.

3.2. Remark (a) If $\mathcal{P}=\left\{s_{1}=t_{1}, \ldots, s_{n}=t_{n}\right\}$ and $\left\{x_{1}, \ldots, x_{k}\right\}$ is the set of all variables which appear in the implication, then the implication

$$
I \equiv(\mathcal{P} \Rightarrow u=v)
$$

is a shorthand for the first-order formula

$$
\left(\forall x_{1}\right) \ldots\left(\forall x_{k}\right)\left(\left(\bigwedge_{i=1}^{n} s_{i}=t_{i}\right) \Rightarrow(u=v)\right) .
$$


Thus a $\Sigma$-algebra $A$ satisfies $I$ iff for every interpretation of variables, i.e., every homomorphism $f: \phi(W) \rightarrow A$, where $W$ contains the variables $x_{1}, \ldots, x_{k}$, we have:

$$
f\left(s_{i}\right)=f\left(t_{i}\right) \text { for } i=1, \ldots, n \text { implies } f(u)=f(v) .
$$

(b) Below we work with a (non specified) finite set $W \subset V$ of variables. Since we always deal with finitely many implications at a time, some set $W$ like that is always sufficient.

3.3. Notation Given an implication

$$
I \equiv(\mathcal{P} \Rightarrow u=v)
$$

we denote by $\sim_{\mathcal{P}}$ the congruence on $\phi(W)$ generated by the equations in $\mathcal{P}$ with the corresponding quotient map

$$
q_{\mathcal{P}}: \phi(W) \rightarrow \phi(W) / \sim_{\mathcal{P}} .
$$

And we denote by $\sim_{I}$ the congruence on $\phi(W)$ generated by the equations in $\mathcal{P} \cup\{u=v\}$ with the corresponding quotient map

$$
q_{I}: \phi(W) \rightarrow \phi(W) / \sim_{I} .
$$

We obtain a quotient map

$$
e_{I}: \phi(W) / \sim_{\mathcal{P}} \longrightarrow \phi(W) / \sim_{I}
$$

such that the triangle

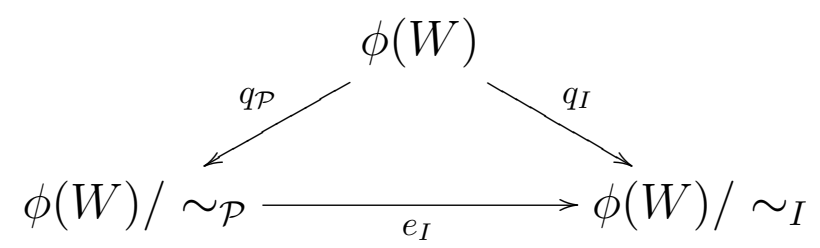

commutes.

3.4. Notation A substitution is a function assigning to every variable a term or, equivalently, a homomorphism $\sigma: \phi(W) \rightarrow \phi(W)$. We write $u^{\sigma}$ instead of $\sigma(u)$, for every equation $u=v$ we denote by $(u=v)^{\sigma}$ the equation $u^{\sigma}=v^{\sigma}$, and we use the notation $\mathcal{P}^{\sigma}=\left\{e^{\sigma}: e \in \mathcal{P}\right\}$.

3.5. Remark It has been first observed by B. Banaschewski and H. Herrlich [8] that a $\sum$-algebra satisfies an implication $I$ iff it is injective w.r.t. the regular epimorphism $e_{I}$. And conversely: for every regular epimorphism $e$ in 
$\operatorname{Alg} \Sigma$ whose domain and codomain are finitely presentable, $e$-injectivity can be expressed by a finite family of implications.

3.6. Deduction System for Implications The deduction system of [20] consists of two axioms

$$
\begin{array}{ll}
\text { Axiom 1: } & \mathcal{P} \Rightarrow u=v \quad \text { if } \mathcal{P} \text { contains } u=v \\
\text { Axiom 2: } & \overline{\mathcal{P} \Rightarrow u=u}
\end{array}
$$

and the following deduction rules

$$
\text { Symmetry: } \quad \frac{\mathcal{P} \Rightarrow u=v}{\mathcal{P} \Rightarrow v=u}
$$

Transitivity: $\quad \frac{\mathcal{P} \Rightarrow u=v, \mathcal{P} \Rightarrow v=w}{\mathcal{P} \Rightarrow u=w}$

Congruence: $\frac{\mathcal{P} \Rightarrow u_{1}=v_{1}, \ldots, \mathcal{P} \Rightarrow u_{n}=v_{n}}{\mathcal{P} \Rightarrow f\left(u_{1}, \ldots, u_{n}\right)=f\left(v_{1}, \ldots, v_{n}\right)}$

for all $n$-ary symbols $f$ in $\Sigma$.

$\begin{array}{ll}\text { Invariance: } & \frac{\mathcal{P} \Rightarrow u=v}{\mathcal{P}^{\sigma} \Rightarrow u^{\sigma}=v^{\sigma}}\end{array}$

for all substitutions $\sigma$.

Cut:

$$
\begin{aligned}
& \mathcal{P} \Rightarrow s_{i}=t_{i}(i=1, \ldots, n),\left\{s_{i}=t_{i}\right\}_{i=1}^{n} \Rightarrow u=v \\
& \mathcal{P} \Rightarrow u=v
\end{aligned}
$$

In all these axioms and rules $u, v$ and $w$, with additional indices and primes, denote arbitrary terms in $\phi(V)$ and $\mathcal{P}$ denotes an arbitrary finite set of equations.

3.7. Remark This deduction system extends naturally Birkhoff's equational logic (consisting of Axiom 2 and the first four deduction rules with $\mathcal{P}=\emptyset$ ). 
3.8. Notation For a given set $E$ of implications and an implication $I$, we write

$$
E \models I
$$

if $I$ is a logical consequence of $E$, i.e., whenever an algebra satisfies all implications in $E$, then it satisfies $I$. And we write

$$
E \vdash I
$$

if there exists a finite proof of $I$ from $E$ using the Deduction System 3.6.

3.9. Lemma If $\mathcal{P}$ is a finite set of equations in $\phi(W)$ then for every pair $u \sim_{\mathcal{P}} v$ of congruent terms we have a proof of $\mathcal{P} \Rightarrow u=v$. Shortly

$$
\vdash(\mathcal{P} \Rightarrow u=v) \text { whenever } u \sim_{\mathcal{P}} v .
$$

The proof is easy.

3.10. Lemma Given homomorphisms

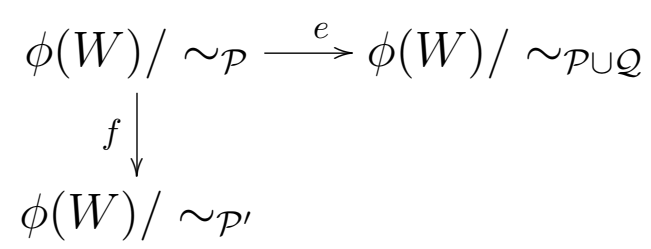

for some finite sets $\mathcal{P}, \mathcal{Q}$ and $\mathcal{P}^{\prime}$ of equations, where e is the canonical quotient morphism, there exists a substitution $\sigma$ with

$$
\mathcal{P}^{\sigma} \text { included in } \sim_{\mathcal{P}^{\prime}}
$$

such that the canonical quotient homomorphism $e^{\prime}: \phi(W) / \sim_{\mathcal{P}^{\prime}} \rightarrow \phi(W) / \sim_{\mathcal{P}^{\prime} \cup \mathcal{P}^{\sigma} \cup \mathcal{Q}^{\sigma}}$ forms a pushout of e along $f$ :

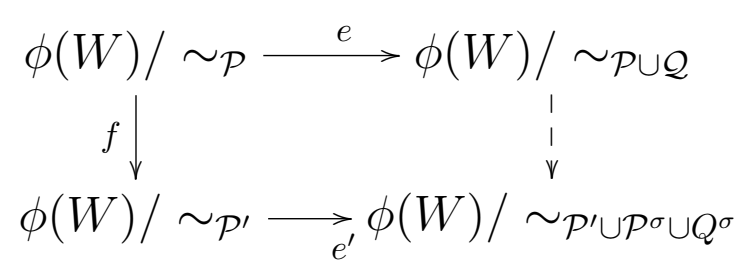

Proof For the given homomorphism

$$
f: \phi(W) / \sim_{\mathcal{P}} \longrightarrow \phi(W) / \sim_{\mathcal{P}^{\prime}}
$$


find a substitution $\sigma$ such that the square

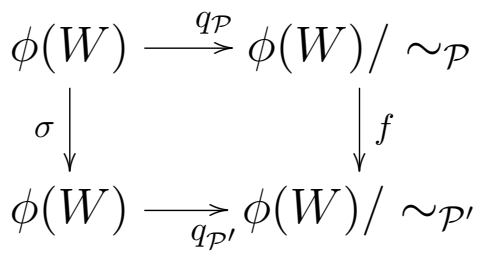

commutes. (To do so, choose a function $i$ splitting $q_{p^{\prime}}$, i.e., $q_{\mathcal{P}^{\prime}} \cdot i=\mathrm{id}$. The morphism

$$
W \stackrel{\eta}{\longrightarrow} \phi(W) \stackrel{q_{\mathcal{P}}}{\longrightarrow} \phi(W) / \sim_{\mathcal{P}} \stackrel{f}{\longrightarrow} \phi(W) / \sim_{\mathcal{P}^{\prime}} \stackrel{i}{\longrightarrow} \phi(W)
$$

has a unique extension to a substitution, i.e., a homomorphism $\sigma: \phi(W) \rightarrow$ $\phi(W)$ such that $\sigma \cdot \eta=i \cdot f \cdot q_{\mathcal{P}} \cdot \eta$. Composed with $q_{\mathcal{P}^{\prime}}$ this yields $\left(q_{\mathcal{P}^{\prime}}\right.$. $\sigma) \cdot \eta=\left(f \cdot q_{\mathcal{P}}\right) \cdot \eta$, thus the square above commutes due to the universal property of $\eta$.) Observe that a pushout of the quotient map $e: \phi(W) / \sim_{\mathcal{P}} \longrightarrow$ $\phi(W) / \sim_{\mathcal{P} \cup \mathcal{Q}}$ along $f$ is a quotient map $q: \phi(W) / \sim_{\mathcal{P}^{\prime}} \longrightarrow \phi(W) / \approx$, where $\approx$ is the smallest congruence containing $\mathcal{P}^{\prime}$ and such that $q \cdot f: \phi(W) / \sim_{\mathcal{P}} \longrightarrow$ $\phi(W) / \approx$ factorizes through $e$. The latter condition is equivalent to saying that given $t, s \in \phi(W)$ with $e \cdot q_{\mathcal{P}}(t)=e \cdot q_{\mathcal{P}}(s)$ then $q \cdot f \cdot q_{\mathcal{P}}(t)=q \cdot f \cdot q_{\mathcal{P}}(s)$. Now $e \cdot q_{\mathcal{P}}$ is the quotient map of $\sim_{\mathcal{P} \cup \mathcal{Q}}$ and $q \cdot f \cdot q_{\mathcal{P}}=q \cdot q_{\mathcal{P}^{\prime}} \cdot \sigma$ where $q \cdot q_{\mathcal{P}^{\prime}}$ is the quotient map of $\approx$. Thus, the latter condition states that

$$
t \sim_{\mathcal{P} \cup \mathcal{Q}} s \text { implies } t^{\sigma} \approx s^{\sigma} .
$$

In other words, $\approx$ is the smallest congruence containing

$$
\mathcal{P}^{\prime} \cup \mathcal{P}^{\sigma} \cup \mathcal{Q}^{\sigma} \text {. }
$$

Thus, a pushout of $e$ and $f$ has the form

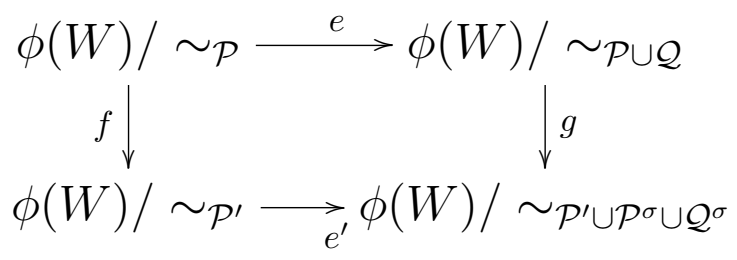

where $e^{\prime}$ is the canonical quotient morphism.

Moreover $\mathcal{P}^{\sigma}$ is included in $\sim_{\mathcal{P}^{\prime}}$ because $q_{\mathcal{P}^{\prime}} \cdot \sigma=f \cdot q_{\mathcal{P}}$ implies that

$$
\text { given } u=v \text { in } \mathcal{P} \text { then } u^{\sigma} \sim_{\mathcal{P}^{\prime}} v^{\sigma} .
$$

3.11. Remark It is well known that the finitely presentable objects of $A \lg \Sigma$ are precisely those isomorphic to the quotient algebras $\phi(W) / \sim_{\mathcal{P}}$ where 
$W \subseteq V$ is a finite set of variables and $\mathcal{P}$ a finite set of equations in $\phi(W)$. And it is easy to verify that, analogously, the epimorphisms between finitely presentable objects are precisely those isomorphic (in the arrow-category) to the canonical quotient maps $e: \phi(W) / \sim_{\mathcal{P}} \rightarrow \phi(W) / \sim_{\mathcal{P} \cup \mathcal{Q}}$ (where $\mathcal{P}$ and $\mathcal{Q}$ are finite sets of equations in $\phi(W))$. The set of all these canonical epimorphisms is denoted by $\mathcal{E}_{f p}$. Taking into account that $\mathcal{E}_{f p}$ is closed under composition and left-cancellable, and using Lemma 3.10, it is obvious that the Completeness Theorem 2.13 remains true if we apply it (instead of to all epimorphisms of $\left.A_{f p}\right)$ just to the set $\mathcal{E}_{f p}$.

3.12. Theorem (see [20]) The deduction system of 3.6 is sound and complete. That is:

$$
E \models I \text { iff } E \vdash I
$$

for every set $E$ of implications and every implication $I$.

Proof It is easy to verify the soundness. Completeness can be derived from Theorem 2.13 by translating 2.12 to the deduction system 3.6. For doing so we are going to work with finite nonempty sets $F$ of implications having the same antecedent,

$$
F=\left\{\mathcal{P} \Rightarrow u_{1}=v_{1}, \ldots, \mathcal{P} \Rightarrow u_{n}=v_{n}\right\} .
$$

We denote by $\mathbb{F}$ the set of all such sets $F$ and put

$$
\mathcal{P}_{F}=\mathcal{P} \text { and } \mathcal{Q}_{F}=\left\{u_{1}=v_{1}, \ldots, u_{n}=v_{n}\right\}
$$

which are finite sets of equations in $\phi(W)$ (for some finite set $W \subseteq V$ of variables).

An implication $I \equiv(\mathcal{P} \Rightarrow u=v)$ is considered as a member of $\mathbb{F}$ by identifying it with the corresponding singleton set $I$. We write

$$
F \vdash G \quad(F, G \in \mathbb{F})
$$

if every member of $G$ can be derived from the finite set $F$ by applying the rules of 3.6.

For every $F \in \mathbb{F}$ we form the canonical epimorphism

$$
e_{F}: \phi(W) / \sim_{\mathcal{P}} \longrightarrow \phi(W) / \sim_{\mathcal{P} \cup \mathcal{Q}}
$$

(where $\mathcal{P}=\mathcal{P}_{F}$ and $\mathcal{Q}=\mathcal{Q}_{F}$, we drop the index $F$ whenever no confusion can arise). This is consistent with Notation 3.3. Let $\mathcal{A}$ be the category of $\Sigma$-algebras and let $\mathcal{A}_{f p}$ be the category of finitely presentable algebras of the 
form $\phi(W) / \sim_{\mathcal{P}}$, where $W \subseteq V$ is a finite set of variables and $\mathcal{P}$ is a finite set of equations (with $\sim_{\mathcal{P}}$ denoting the congruence generated by $\mathcal{P}$ ). By Theorem 2.13 and Remark 3.11, the Finitary Injectivity Deduction System is complete for epimorphisms in $\mathcal{E}_{f p}$, and we will use this completeness to prove the present theorem by verifying the following:

(A) an application of the rules of 2.12 to morphisms $e_{F_{1}}, \cdots, e_{F_{n}}\left(F_{i} \in \mathbb{F}\right)$ always lead to a conclusion of the form $e_{F}(F \in \mathbb{F})$ with

$$
\bigcup_{i=1}^{n} F_{i} \vdash F
$$

and

(B) if $e_{F}=e_{F^{\prime}}\left(F, F^{\prime} \in \mathbb{F}\right)$ then $F \vdash F^{\prime}$.

By proving (A) and (B), the completeness of 3.6 follows: given a set $E$ of implications with a logical consequence $I$,

$$
E \models I
$$

we know from Remark 3.5 that the injectivity w.r.t. $e_{I}$ is a logical consequence of the injectivity w.r.t. $\hat{E}=\left\{e_{\hat{I}}, \hat{I} \in E\right\}$. By the Completeness Theorem 2.13 we conclude that a formal proof of $e_{I}$ from $\hat{E}$ exists in the deduction system 2.12 , that is, there are implications $I_{1}, \cdots, I_{n} \in E$ such that $e_{I_{1}}, \cdots, e_{I_{n}} \vdash e_{I}$ in Finitary Injectivity Deduction System. Due to (A), every step in that proof is of the form $e_{F}$ for some $F$ such that $\left\{I_{1}, \cdots, I_{n}\right\} \vdash F$. In particular, the last line, $e_{I}$, is equal to some such $e_{F}$, which by $(\mathrm{B})$ implies $F \vdash I$. Consequently, we obtain $\left\{I_{1}, \cdots, I_{n}\right\} \vdash I$, and thus $E \vdash I$, as required.

The statement (B) follows immediately from 3.9.

Proof of (A). Our task is to prove for every rule of 2.12 that if the premises have the form $e_{F_{1}}, \cdots, e_{F_{n}}$ then the conclusion has the form $e_{F}$ where $\cup F_{i} \vdash$ $F$.

We proceed by inspecting the rules individually.

(1) IDEnTity: Suppose that $e_{F}$ is an identity morphism. Then the two congruences $\sim_{\mathcal{P}}$ and $\sim_{\mathcal{P} \cup \mathcal{Q}}$ coincide, thus for each $u=v$ in $\mathcal{Q}$ we have

$$
u \sim_{\mathcal{P}} v
$$


and Lemma 3.9 gives us $\vdash \mathcal{P} \Rightarrow u=v$.

(2) COMposition: Let $e_{F}$ and $e_{F^{\prime}}$ be two morphisms in $\mathcal{E}_{f p}$ which compose, with $F$ and $F^{\prime}$ members of $\mathbb{F}$ :

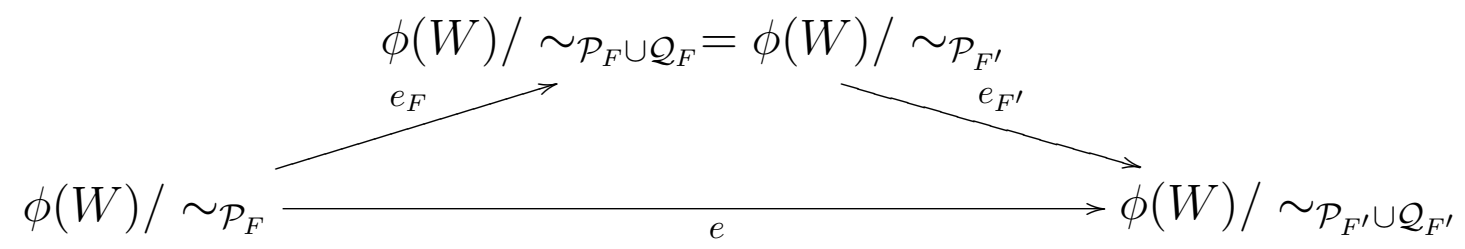

Since $\mathcal{P}_{F} \cup \mathcal{Q}_{F}$ generates the same congruence as $\mathcal{P}_{F^{\prime}}$, it follows that $\mathcal{P}_{F} \cup$ $\mathcal{Q}_{F} \cup \mathcal{Q}_{F^{\prime}}$ generates the same congruence as $\mathcal{P}_{F^{\prime}} \cup \mathcal{Q}_{F^{\prime}}$, consequently,

$$
e=e_{F^{\prime \prime}}
$$

for

$$
F^{\prime \prime}=F \cup\left\{\mathcal{P}_{F} \Rightarrow u=v ; u=v \text { in } \mathcal{Q}_{F^{\prime}}\right\} .
$$

It is our task to prove that

$$
F \cup F^{\prime} \vdash F^{\prime \prime}
$$

That is, given $u=v$ in $\mathcal{Q}_{F^{\prime}}$, we are going to derive the implication $\mathcal{P}_{F} \Rightarrow$ $u=v$ from $F \cup F^{\prime}$.

Using Lemma 3.9 on any $s=t$ in $\mathcal{P}_{F^{\prime}}$, we get

$$
\vdash \mathcal{P}_{F} \cup \mathcal{Q}_{F} \Rightarrow s=t
$$

therefore, by Cut,

$$
F \vdash\left(\mathcal{P}_{F} \Rightarrow s=t\right) \quad\left(\text { for all } s=t \text { in } \mathcal{P}_{F^{\prime}}\right) .
$$

Since for $u=v$ in $\mathcal{Q}_{F^{\prime}}$ we have, trivially,

$$
F^{\prime} \vdash\left(\mathcal{P}_{F^{\prime}} \Rightarrow u=v\right)
$$

we conclude, again by Cut, that

$$
F \cup F^{\prime} \vdash\left(\mathcal{P}_{F} \Rightarrow u=v\right)
$$

as requested.

(3) CANCELlation: We are given a commutative diagram

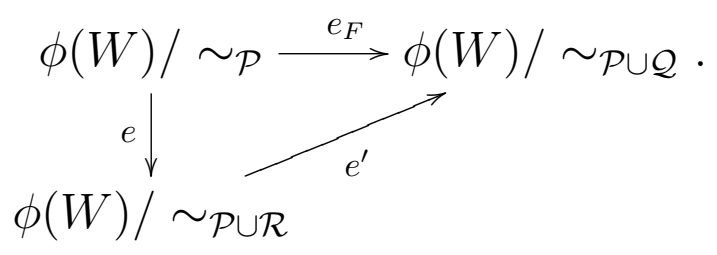


Then $e=e_{F^{\prime}}$ for

$$
F^{\prime}=\{\mathcal{P} \Rightarrow u=v ; u=v \text { in } \mathcal{R}\} .
$$

We now have to prove that $F \vdash F^{\prime}$, i.e., for every $u=v$ in $\mathcal{R}$ we have to verify

$$
F \vdash \mathcal{P} \Rightarrow u=v .
$$

Since $e_{F}=e^{\prime} \cdot e_{F^{\prime}}$, we conclude $u \sim_{\mathcal{P} \cup \mathcal{Q}} v$, thus, by Lemma 3.9,

$$
\vdash \mathcal{P} \cup \mathcal{Q} \Rightarrow u=v
$$

Therefore, Cut yields

$$
F \vdash \mathcal{P} \Rightarrow u=v
$$

as requested.

(4) Pushout: Let

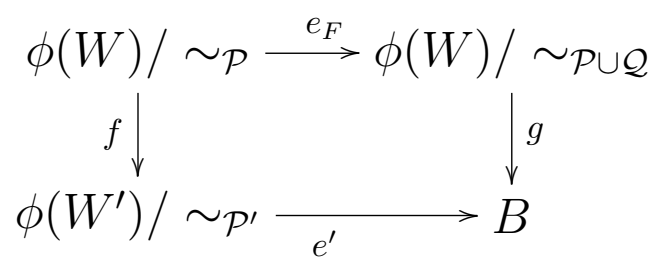

be a pushout where $F \in \mathbb{F}$ (and we put $\mathcal{P}_{F}=\mathcal{P}$ and $\mathcal{Q}_{F}=\mathcal{Q}$ ), and $e^{\prime} \in \mathcal{E}_{f p}$. By Lemma 3.10 we have a substitution $\sigma$ with $\mathcal{P}^{\sigma}$ included in $\sim_{\mathcal{P}^{\prime}}$, and

$$
e^{\prime}=e_{F^{\prime}} \text { for } F^{\prime}=\left\{\mathcal{P}^{\prime} \Rightarrow u^{\sigma}=v^{\sigma} ; u=v \text { in } \mathcal{P} \cup \mathcal{Q}\right\} .
$$

Thus, we need to show that $F \vdash F^{\prime}$. First for every $u=v$ in $\mathcal{P}$ we have

$$
\vdash \mathcal{P}^{\prime} \Rightarrow u^{\sigma}=v^{\sigma}
$$

since $u=v$ in $\mathcal{P}$ implies $u^{\sigma} \sim_{\mathcal{P}^{\prime}} v^{\sigma}$, see Lemma 3.9. Secondly, for every $s=t$ in $\mathcal{Q}$ we verify

$$
F \vdash \mathcal{P}^{\prime} \Rightarrow s^{\sigma}=t^{\sigma} .
$$

In fact, from Invariance in 3.6 we know that

$$
F \vdash \mathcal{P}^{\sigma} \Rightarrow s^{\sigma}=t^{\sigma}
$$

and since $\vdash \mathcal{P}^{\prime} \Rightarrow u^{\sigma}=v^{\sigma}$ (for all $u^{\sigma}=v^{\sigma}$ in $\mathcal{P}^{\sigma}$ ), this yields by Cut the desired statement $F \vdash \mathcal{P}^{\prime} \Rightarrow s^{\sigma}=t^{\sigma}$. Consequently, $F \vdash F^{\prime}$. 


\section{Implications in Coalgebra}

4.1. Assumption Throughout this section we work with coalgebras over a functor $H$ : Set $\rightarrow$ Set. We assume that $H$ is accessible, i.e., there exists an infinite cardinal $k$ such that $H$ preserves $k$-filtered colimits. More detailed, we say that $H$ is $k$-accessible.

A coalgebra is a pair $(A, \alpha)$ where $A$ is a set and $\alpha: A \rightarrow H A$ is a function. Homomorphisms from $(A, \alpha)$ to another coalgebra $(B, \beta)$ are the functions $f: A \rightarrow B$ with $\beta \cdot f=H f \cdot \alpha$. We interpret coalgebras as systems with a set $A$ of states and a set $H A$ of possible observations we make. And homomorphisms express functions simulating the observations in a different system.

We denote by Coalg $H$ the category of coalgebras and homomorphisms.

4.2. Examples (see [22]) A sequential automaton with a state set $A$ and input set $I$ can be viewed as a coalgebra of the functor

$$
H=(-)^{I} \times\{t t, f f\}
$$

In fact, a sequential automaton is specified by the next-state map $A \times I \rightarrow A$ which in the curried form yields $\delta: A \rightarrow A^{I}$, and by the predicate "accepting state" given by $a: A \rightarrow\{t t, f f\}$. This defines a coalgebra

$$
<\delta, a>: A \rightarrow A^{I} \times\{t, f f\}
$$

(ii) The coalgebras over

$$
H X=X \times X+1
$$

are dynamic systems with binary input $\{0,1\}$ and with deadlock states (not reacting to input). Given a state set $A$, the coalgebra structure

$$
\alpha: A \rightarrow A \times A+1
$$

maps deadlock states to the right-hand summand, and non-deadlock states to the pair of next states corresponding to input 0 and 1, respectively.

(iii) Generalizing the previous examples, let $\Sigma$ be a signature (possibly infinitary) and let $H_{\Sigma}$ be the corresponding polynomial functor

$$
H_{\Sigma} X=\coprod_{\sigma \in \Sigma} X^{n} \quad n=\text { arity of } \sigma .
$$

A coalgebra is given by a set $A$ of states and an assignment $\alpha$ which to every state $a$ yields an output $\sigma \in \Sigma$ and an $n$-tuple (of "next states") for $n=\operatorname{ar}(\sigma)$. 
(iv) The finite-power-set functor $\mathcal{P}_{\text {fin }}$ has as coalgebras all finitely branching graphs $A$. Here $\alpha: A \rightarrow \mathcal{P}_{\text {fin }} A$ assigns to every node the set of all neighbor nodes. Coalgebra homomorphisms $f: A \rightarrow B$ are those graph homomorphisms such that for every node $a \in A$ and every edge $f(a) \rightarrow b$ in $B$ there exists an edge $a \rightarrow a^{\prime}$ in $A$ with $b=f\left(a^{\prime}\right)$.

4.3. Notation For every cardinal $k$ we denote by $C(k)$ a cofree coalgebra on $k$ colors. Thus, considering the cardinal $k$ (as usual) as the set of all smaller ordinals, $C(k)$ is given by a coalgebra structure

$$
\tau: C(k) \rightarrow H C(k)
$$

and a coloring

$$
\varepsilon: C(k) \rightarrow k
$$

universal in the following sense:

For every coalgebra $A$ and every coloring $f: A \rightarrow k$

there exists a unique homomorphism $f^{\sharp}: A \rightarrow C(k)$ such that $f=\varepsilon \cdot f^{\sharp}$.

4.4. Examples (i) If $k=1$ then $C(1)$ is simply a terminal coalgebra which we denote by

$$
\tau: T \rightarrow H T
$$

In the example of sequential automata we have

$$
T=\mathcal{P} I^{\star} \quad \text { (the set of formal languages) }
$$

with

$$
\tau: \mathcal{P} I^{\star} \rightarrow\left(\mathcal{P} I^{\star}\right)^{I} \times \text { bool }
$$

given by the automaton structure of $\mathcal{P} I^{\star}$ where a formal language $L \subseteq I^{\star}$ is accepting iff it contains the empty word, and the reaction of $L$ to an input $i \in I$ is the Brzozowski derivative $\left\{u \in I^{\star} ; i u \in L\right\}$.

For every sequential automaton $A$ the unique coalgebra homomorphism $f^{\sharp}: A \rightarrow T$ assigns to every state $a$ the language $f^{\sharp}(a) \subseteq I^{\star}$ accepted by $A$ with the initial state $a$.

(ii) A terminal coalgebra of $H_{\Sigma}$ is the coalgebra $T_{\Sigma}$ of all $\Sigma$-trees, that is, trees labelled in $\Sigma$ so that a node with an $n$-ary label has precisely $n$ children. More generally: the cofree coalgebra on $k$ colors

$$
C_{\Sigma}(k)
$$


consists of all $\Sigma$-trees with an additional coloring of nodes by $k$ colors. Here

$$
\tau: C_{\Sigma}(k) \rightarrow \coprod_{\sigma \in \Sigma} C_{\Sigma}(k)^{n}
$$

assigns to a tree $t$ whose root is labelled by an $n$-ary operation $\sigma \in \Sigma$ and has color $i \in k$ the $n$-tuple of the maximum subtrees of $t$ in the $\sigma$-summand $C_{\Sigma}(k)^{n}$, whereas $\varepsilon: C_{\Sigma}(k) \rightarrow k$ maps $t$ to the colors of its root,

$$
\varepsilon(t)=i \text {. }
$$

For example the functor $H=X \times X+1$ of $4.2\left(\right.$ ii) has $C_{\Sigma}(k)$ equal to all $k$-colored trees such that each node has zero or two children.

4.5. Remark (i) Every accessible functor has cofree coalgebras. In other words, the canonical forgetful functor from Coalg $H$ to Set is a left adjoint. See [9].

(ii) If $H$ preserves $k$-filtered colimits then the cofree coalgebra $C(k)$ was used by Jan Rutten [22] for presentation of classes of coalgebras precisely dual to equational presentation of algebras (via quotients of a free algebra on $k$ generators):

4.6. Definition [22] For a $k$-accessible functor $H$ a coequation is a subset $m: M \hookrightarrow C(k)$ of a cofree coalgebra on $k$ colors. A coalgebra $A$ satisfies the coequation iff for every coloring $f$ of $A$ by $k$ colors the homomorphism $f^{\sharp}$ factors through the subobject

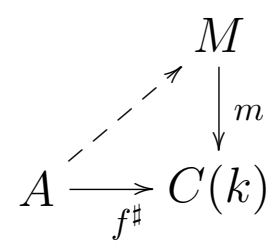

4.7. Theorem [22] For a class $\mathcal{A}$ of coalgebras the following statements are equivalent:

(i) $\mathcal{A}$ is closed under coproducts, subcoalgebras and quotients and

(ii) $\mathcal{A}$ can be presented by a coequation.

4.8. Examples (i) All sequential automata such that from each state a final state is reachable (in finitely many steps) are presented by the one color coequation

$$
\mathcal{P} I^{\star}-\{\emptyset\} \hookrightarrow \mathcal{P} I^{\star}
$$


The coequation $\{L ; \varepsilon \in L\} \hookrightarrow \mathcal{P} I^{\star}$, where $\varepsilon$ denotes the empty word, presents all automata where each state is accepting.

(ii) Given a coalgebra $A$ of the polynomial functor $H_{\Sigma}$, see 4.2(iii), all states mapped by $\alpha$ to the summand of $H_{\Sigma} A$ corresponding to a nullary operation are called deadlock states. The coequation

$$
\{t ; t \text { is a finite colored } \Sigma \text {-tree }\} \hookrightarrow C_{\Sigma}(k)
$$

presents all coalgebras without deadlock states.

(iii) For the dynamic systems of example 4.2(ii) the coequation

$$
C_{\Sigma}(k)-\{t\} \hookrightarrow C_{\Sigma}(k)
$$

where $t$ is the tree

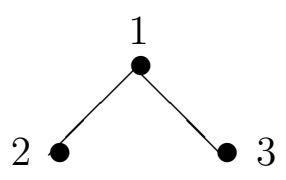

presents all subsystems $A$ such that if a state $a$ reaches deadlock states by both inputs, then those deadlock states are equal.

4.9. Remark A logic of coequations has been presented in [1]. We now generalize this to implications, as introduced by Peter Gumm:

4.10. Definition (see [14]) By an implication for coalgebras is meant an expression

$$
M \Rightarrow N
$$

where

$$
N \hookrightarrow M \hookrightarrow C(k)
$$

are subsets of the free coalgebra. A coalgebra $A$ is said to satisfy the implication $M \Rightarrow N$ provided that for coloring $f: A \rightarrow k$ such that $f^{\sharp}$ factors through $m$ it follows that $f^{\sharp}$ factors through $m \cdot h$ :

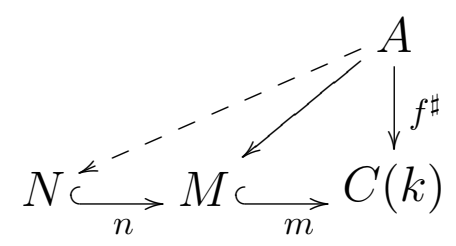

4.11. Example For sequential automata, the implication

$$
\{L ; \varepsilon \in L\} \Rightarrow\{\{\varepsilon\}\}
$$


presents all automata which either have a non-final state, or there are no transitions (from a state to a different one).

4.12. Theorem [2] For a class $\mathcal{A}$ of coalgebras the following statements are equivalent:

(i) $\mathcal{A}$ is closed under coproducts and quotients and

(ii) $\mathcal{A}$ can be presented by a collection of implications.

4.13. Notation For every coequation $m: M \hookrightarrow C(k)$ we denote by

(i) $\hat{m}: \hat{M} \hookrightarrow C(k)$ the largest subcoalgebra of $C(k)$ contained in $m$ : $M \hookrightarrow C(k)$ and

(ii) $m^{\sigma}: M^{\sigma} \hookrightarrow C(k)$, where $\sigma: C(k) \rightarrow C(k)$ is an endomorphism of the cofree coalgebra, the inverse image of $M$ under $\sigma$.

4.14. Remark (i) As proved in [15], every subset $M$ has a largest subcoalgebra contained in it.

The passage from $M$ to $\hat{M}$ is dual to the passage, used in universal algebra, from an equivalence relation $\sim$ on a free algebra to the congruence that $\sim$ generates.

(ii) The passage from $M$ to $M^{\sigma}$ is dual to the passage, used in universal algebra, from a set of equations to that set obtained via a given substitution $x \mapsto \sigma(x)$ for all variables $x \in X$; here $\sigma$ is an endomorphism of $F(X)$.

4.15. Example For $H=H_{\Sigma}$, the subcoalgebra $\hat{M}$ consists precisely of all trees $t \in M$ such that every subtree of $t$ lies in $M$. And $M^{\sigma}$ is the set of all trees in whose recoloring via a given coloring $C_{\Sigma}(k) \rightarrow k$ (which is equivalent to giving an endomorphism $\sigma$ of $\left.C_{\Sigma}(k)\right)$ yields a tree in $M$, see [1].

4.16. Lemma If $N \subseteq M$ and $\hat{M}=\hat{N}$ then the implication $M \Rightarrow N$ is satisfied by any coalgebra.

Proof As proved in [4], the image of a coalgebra homomorphism $h: A \rightarrow B$ is always a subcoalgebra of $B$.

Let $M$ and $N$ be under the above conditions, and, for $A$ a coalgebra, consider a coloring $f: A \rightarrow k$ with $f^{\sharp}[A] \subseteq M$. Since $f^{\sharp}[A]$ is a subcoalgebra of $C(k)$, it is contained in $\hat{M}$, then also in $N$, because $\hat{M}=\hat{N} \subseteq N$. 
4.17. Notation For every implication $h \equiv(M \Rightarrow N)$, the subcoalgebra $\hat{N}$ is (due to $N \subseteq M$ ) contained in the subcoalgebra $\hat{M}$ and we denote by

$$
\hat{h}: \hat{N} \rightarrow \hat{M}
$$

the homomorphism which is the codomain restriction of the embedding $\hat{N} \hookrightarrow$ $C(k)$.

The following corollary is easily derived from 4.16.

4.18. Corollary A coalgebra satisfies an implication $h \equiv(M \Rightarrow N)$ iff it is projective w.r.t. $\hat{h}$.

4.19. Remark (i) The homomorphism $\hat{h}$ is a regular monomorphism. In fact, regular monomorphisms in Coalg $H$ are precisely the homomorphisms which are one-to-one functions, i.e. monomorphisms in Set, see [16].

(ii) We thus can apply the deduction system 2.2 to the category $(\text { Coalg } H)^{o p}$. In contrast, the finitary deduction system 2.12 is not relevant here since in general $(\text { Coalg } H)^{o p}$ does not have nontrivial finitely presentable objects. In fact, if $H$ is the constant functor with value 1 then Coalg $H=$ Set and in $(\text { Set })^{o p}$ no object of more than one element is finitely presentable.

(iii) An implication $M \Rightarrow N$ is called a consequence of a set $\mathcal{H}$ of implications provided that, given a coalgebra $A$ satisfying every implication in $\mathcal{H}$, then $A$ satisfies $M \Rightarrow N$. Example: $\hat{M} \Rightarrow \hat{N}$ is a consequence of $M \Rightarrow N$.

(iv) We now reformulate the deduction system 2.2 in the languague of implications:

4.20. Definition The Deduction System for Coalgebraic Implications consists of the following deduction rules

$$
\begin{aligned}
& \text { (1) } \overline{M \Rightarrow M} \\
& \text { (2) } \begin{array}{l}
M \Rightarrow N \quad N \Rightarrow P \\
\hline M P
\end{array} \\
& \text { (3) } \frac{M \Rightarrow N}{P \Rightarrow Q} \quad \text { if } \hat{M}=\hat{P}, \hat{N}=\hat{Q} \text { and } Q \subseteq P \\
& \text { (4) } \begin{array}{l}
M \Rightarrow N_{i} \quad(i \in I) \\
M \Rightarrow \bigcap_{i \in I} N_{i}
\end{array}
\end{aligned}
$$




$$
\begin{array}{lll}
\text { (5) } & \frac{M \Rightarrow N}{M \Rightarrow N \cup N_{0}} & \text { if } N_{0} \subseteq M \\
& & \\
\text { (6) } & \frac{M \Rightarrow N}{M \cap P \Rightarrow N \cap P} & \\
& & \\
\text { (7) } & M \Rightarrow N & \text { for all homomorphisms } \\
& M^{\sigma} \Rightarrow N^{\sigma} & \sigma: C(k) \rightarrow C(k)
\end{array}
$$

4.21. Remark The soundness of the rule (3) is Corollary 4.18. All other rules, possibly with the exception of (7), are obviously sound. To verify (7), let $A$ be a coalgebra satisfying $M \Rightarrow N$ for subsets

$$
N \hookrightarrow M \hookrightarrow C(k) .
$$

Recall that $(-)^{\sigma}$ denotes the preimage under the given homomorphism $\sigma$ : $C(k) \rightarrow C(k)$. Thus, in the following diagram

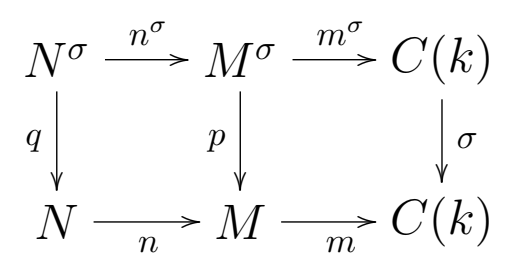

the right-hand square and the outer square are pullbacks in Set. Consequently, the left-hand square is a pullback in Set too.

Suppose that the coalgebra $A$ satisfies $M \Rightarrow N$. Let $f: A \rightarrow k$ be a coloring with $f^{\sharp}[A] \subseteq M^{\sigma}$. Then $f^{\sharp}$ has a codomain restriction to a homomorphism $u: A \rightarrow M^{\sigma}:$

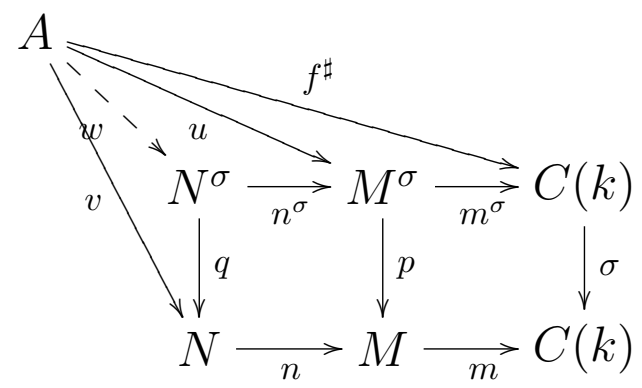

For the coloring $\varepsilon \cdot \sigma \cdot f^{\sharp}: A \rightarrow k$, the corresponding homomorphism is $g^{\sharp}=\sigma \cdot f^{\sharp}$, and we see that $p \cdot u: A \rightarrow M$ is its codomain restriction. Thus, 
$g^{\sharp}[A] \subseteq M$, which implies that $g^{\sharp}[A] \subseteq N$. Let $v: A \rightarrow N$ be the codomain restriction of $g^{\sharp}$. Then, the universal property of the left-hand pullback above yields $w: A \rightarrow N^{\sigma}$ with $f^{\sharp}=m^{\sigma} \cdot n^{\sigma} \cdot w$. That is $f^{\sharp}[A] \subseteq N^{\sigma}$. Therefore, $A$ satisfies $M^{\sigma} \Rightarrow N^{\sigma}$.

4.22. Remark Recall that a functor is said to preserve inverse images provided it preserves pullbacks of monomorphisms along any morphism. This is a usual property of set functors: $H_{\Sigma}, \mathcal{P}$ and $\mathcal{P}_{\text {fin }}$ are examples of functors preserving inverse images. And any composite, product, coproduct and subfunctor "inherits" the property of preserving inverse images. It is easy to verify that if $H$ preserves inverse images then the forgetful functor Coalg $H \rightarrow$ Set lifts them.

4.23. Completeness Theorem Let $H$ be an accessible functor preserving inverse images. Then the deduction system 4.20 is complete: every consequence of a set of implications has a proof from that set.

\section{Proof Let}

$$
k \equiv(M \Rightarrow N)
$$

be a consequence of a set $\mathcal{H}$ of implications. By 4.18, the morphism $\hat{k}: \hat{N} \hookrightarrow$ $\hat{M}$ is, in $(\text { Coalg } H)^{o p}$, an injectivity consequence of the set

$$
\hat{\mathcal{H}}=\{\hat{h}\}_{h \in \mathcal{H}} .
$$

By 2.5, we have a proof of $\hat{k}$ from $\hat{\mathcal{H}}$ using the deduction system 2.2 in its dual form. If $\alpha$ is the length of the proof and the line $i$ is the formula $f_{i}(i \leq \alpha)$, we have that

$$
f_{\alpha}=\hat{k} \text {. }
$$

We now translate this proof into a proof of $k$ from $\mathcal{H}$ in the deduction system 4.20. Our translation will be such that, whenever a part $f_{i}(i \leq \beta)$ has been already translated, then for every line

$$
f_{j}: P \rightarrow Q \quad \text { where } j \leq \beta
$$

the translation contains the line

$$
Q \Rightarrow P \text {. }
$$

This will finish the proof: at the end of the translation the last line $f_{\alpha}: \hat{N} \rightarrow$ $\hat{M}$ guarantees that one line of the translated proof is $\hat{M} \Rightarrow \hat{N}$. We then 
add the line $M \Rightarrow N$ (using Rule (3)), thus concluding the formal proof of $M \Rightarrow N$ from $\mathcal{H}$.

The translation is performed by transfinite induction: given $\beta \leq \alpha$ such that the preceding lines $f_{i}(i<\beta)$ have already been translated, we translate $f_{\beta}$ as follows.

(i) If $f_{\beta}=\hat{h}: \hat{Q} \rightarrow \hat{P}$ is one of the assumptions, where $h \equiv(P \Rightarrow Q)$ lies in $\mathcal{H}$, we translate $f_{\beta}$ by two lines:

$$
\begin{gathered}
P \Rightarrow Q \quad(\text { assumption in } \mathcal{H}) \\
\hat{P} \Rightarrow \hat{Q} \quad \text { (by Rule }(3))
\end{gathered}
$$

(This second line is needed for our assumption about the translation, see above.)

(ii) If $f_{\beta}=i d_{M}$ our translation is

$$
M \Rightarrow M \quad \text { by Rule (1) }
$$

(iii) If $f_{\beta}=f_{i} \cdot f_{j}$ for some $i, j$ smaller than $\beta$

$$
P \stackrel{f_{j}}{\longrightarrow} Q \stackrel{f_{i}}{\longrightarrow} R
$$

then our translation contains $Q \Rightarrow P$ and $R \Rightarrow Q$ and we translate $f_{\beta}$ as

$$
R \Rightarrow P \quad \text { by Rule }(2) \text {. }
$$

(iv) If $f_{\beta}: P \rightarrow Q$ is an intersection of the preceding lines $f_{i(t)}: P_{i(t)} \rightarrow Q$ for $t \in T$, then our translation contains the implications $Q \Rightarrow P_{i(t)}$, corresponding to these lines, and $P=\cap_{t \in T} P_{i(t)}$. Thus, our translation of $f_{\beta}$ is

$$
Q \Rightarrow P \quad \text { by Rule (4). }
$$

(v) If $f_{\beta}$ is obtained by right cancellation:

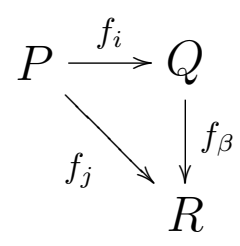

for some $i, j$ smaller than $\beta$, then our translation contains $R \Rightarrow P$, and we see that $P \subseteq Q$. Therefore, we translate $f_{\beta}$ as 


$$
R \Rightarrow Q \quad \text { by Rule (5) }
$$

(vi) If $f_{\beta}$ is obtained from a line $f_{i}(i<\beta)$ via a pullback

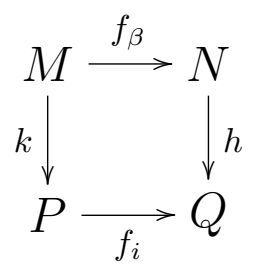

we decompose $h$ as an epimorphism $e: N \rightarrow Q^{\prime}$ followed by a monomorphism $u: Q^{\prime} \rightarrow Q$ and form the appropriate pullbacks

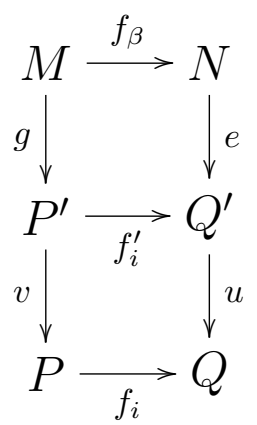

We start the translation of $f_{\beta}$ by writing

$$
Q^{\prime} \Rightarrow P^{\prime}
$$

by Rule (6) applied to $Q \Rightarrow P$ via the intersection with $Q^{\prime}$. Next, express $N$ and $Q^{\prime}$ as subcoalgebras of $C(k)$

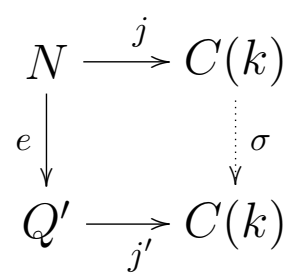

and find a homomorphism $\sigma: C(k) \rightarrow C(k)$ with the above square commuting. This is trivial if $N=\emptyset$. Assuming that $N \neq \emptyset$, we choose $u: C(k) \rightarrow N$ with $u \cdot j=i d$ and we extend $j \cdot e \cdot u$ to an homomorphism $\sigma$ satisfying

$$
\varepsilon \cdot \sigma=\varepsilon \cdot j^{\prime} \cdot e \cdot u \text {. }
$$

Therefore,

$$
\varepsilon \cdot(\sigma \cdot j)=\varepsilon \cdot\left(j^{\prime} \cdot e\right)
$$


Since $\sigma \cdot i$ and $j^{\prime} \cdot e$ are homomorphisms, the last equation implies that the square above commutes. We conclude that

$$
Q^{\prime}=\sigma[N] \text { and } M=\sigma^{-1}\left[P^{\prime}\right]
$$

since $e$ is an epimorphism.

We continue the translation of $f_{i}$ : from $\sigma[N]=Q^{\prime} \Rightarrow P^{\prime}$ we derive

$$
\sigma^{-1} \sigma[N] \Rightarrow \sigma^{-1}\left(P^{\prime}\right)=M \quad \text { by Rule }(7) \text {. }
$$

Consequently

$$
\sigma^{-1} \sigma[N] \cap N \Rightarrow M \cap N \quad \text { by Rule (6) }
$$

and, since $M \subseteq N \subseteq \sigma^{-1} \sigma[N]$, we conclude

$$
N \Rightarrow M
$$

\section{References}

[1] J. Adámek, A Logic of coequations, Lect. Notes Comput. Sci. 3464, Springer-Verlag 2005, 70-86.

[2] J. Adámek, The intersection of algebra and coalgebra, to appear in Theoret. Comput. Sci.

[3] J. Adámek, H. Herrlich and G. Strecker, Abstract and Concrete Categories, John Wiley, New York 1990.

[4] J. Adámek, H.-E. Porst, On varieties and covarieties in a category, Mathem. Str. Comput. Sci. 13 (2003), 201-232.

[5] J. Adámek, H.-E. Porst, On tree coalgebras and coalgebra presentations, Theoret. Comput. Sci. 311 (2004) 257-283.

[6] M.A. Arbib, E.G. Manes, Parametrized data types do not need highly constrained parameters, Inform. and Control 52 (1982), 130-158.

[7] S. Awodey, J. Hughes, Modal operators and the formal dual of Birkoff's completeness theorem, Mathem. Struct. in Comput. Sci. 13 (2003), 233-258.

[8] B. Banaschewski and H. Herrlich, Subcategories defined by implications, Houston J. Math. 2 (1976), 149-171.

[9] M. Barr, Terminal coalgebras in well-founded set theory, Theoret. Comput. Sci. 124 (1984), 182-192.

[10] G. Birkhoff, On the structure of abstract algebras, Proceedings of the Cambridge Philosophical Society 31 (1935), 433-454.

[11] P. Gabriel, M. Zisman, Calculus of Fractions and Homotopy Theory, Springer, 1967.

[12] H.P. Gumm, Elements of the general theory of coalgebras, preprint 1999.

[13] H.P. Gumm, Birkhoff's variety theorem for coalgebras, Contributions to General Algebra 13 (2000), 159-173.

[14] H.P. Gumm, Equational and implicational classes of coalgebras, Theoret. Comput. Sci. 260 (2001), 57-69.

[15] H.P. Gumm, T. Schröder, Covarieties and complete covarieties, Electron. Notes in Theoret. Comput. Sci. 11 (1998). 
[16] H.P. Gumm, T. Schröder, Coalgebraic structure from weak limit preserving functors, Electron. Notes in Theoret. Comput. Sci. 32 (2000).

[17] M. Hébert, K-purity and orthogonality, Theory Appl. Categ. 12 (2004), 355-371.

[18] M. Hébert, J. Adámek and J. Rosický, More on orthogonality in locally presentable categories, Cah. Topol. Géom. Différ. Catég. 42 (2001), 51-80.

[19] B. Jacobs, The temporal logic of coalgebras via Galois algebras, Mathem. Struct. in Comput. Sci. 11 (2002), 875-903.

[20] R. Quackbenbush, Completeness theorems for universal algebra and implicational logics of algebras via congruences, Proc. Amer. Math. Society 103 (1988) 1015-1021.

[21] G. Roşu, Complete Categorical Equational Deduction, Lect. Notes in Comput. Sci. 2142 (2001), 528-538.

[22] J. Rutten, Universal Coalgebra: a theory of systems, Theoret. Comput. Sci. 249 (2000), 3-80.

[23] L. Sousa, Solid hulls of concrete categories, Appl. Categ. Structures 3 (1995), 105-118.

[24] J. Worrell, On the final sequence of a finitary set functor, Theoret. Comput. Sci. 338 (2005), 184-199.

JiŘí ADÁMEK

Department of Theoretical Computer Science, Technical University of Braunschweig, Postfach 3329, 38023 Braunschweig, Germany

Manuela Sobral

Departamento de Matemática da Universidade de Coimbra, Apartado 3008, 3000 Coimbra, PoRTugal

LuRdes SOUSA

Departamento de Matemática da Escola Superior de Tecnologia de Viseu, Campus Politécnico, 3504-510 Viseu, Portugal 